\title{
The Association of Clinical Pathologists: 91st general meeting
}

The 91st general meeting was held at Imperial College, London, from 19 to 21 September 1973. Abstracts of the scientific communications follow. The Presidential Address, 'Haematology-trends and opportunities', given by Professor E. OK. Blackburn, and the Dyke Foundation Lecture, 'Men and machines', given by Professor N. H. Martin, are publishedEin the November issue.

Giant Nuclear Masses in the Lungs and Blood in Malignant Malaria

H. B. GOODALl (Royal Infirmary, Dundee) Numerous large naked nuclear masses have been found in the lungs of two patients dead from malignant (subtertian, falciparum) malaria. These structures are probably derived from vascular endothelial cells damaged and shed in the process of disseminated intravascular coagulation which is the pathogenetic mechanism causing the complications of malignant malaria, particularly the cerebral damage. Ten buffy coat smears and 30 thick films of blood from one of these patients taken during the terminal illness all contain nuclear masses. Microscopy of such simple preparations, of quantitative modifications of them, or of more elaborate cell concentrates, appears to offer a new method for the detection of disseminated intravascular coagulation in malaria, a method to supplement current diagnostic tests, eg, the estimation of fibrin degradation products.

Clinical and Diagnostic Importance of Synovial Fluid Examination

B. VERNON-ROBERTS (The London Hospital Medical College, London) The examination of a sample of synovial fluid from a joint effusion can establish or exclude the diagnosis of crystal synovitis or septic arthritis; it can often provide sufficient evidence for a definite diagnosis in effusions of uncertain aetiology; and it can indicate the degree of disease activity in rheumatoid arthritis and other inflammatory arthropathies.

In the normal knee joint there is less than $3 \mathrm{ml}$ of clear, pale yellow, highly viscous fluid. It contains less than 750 leucocytes $/ \mathrm{mm}^{3}$. The majority of cells are small lymphocytes, and the remainder are neutrophil polymorphs and mononuclear phagocytes.
In osteoarthrosis the fluid is usually increased in amount, but remains clear and often retains its high viscosity. The total and differential cell count seldom differs from the normal.

In rheumatoid arthritis the fluid is increased in amount, is cloudy due to increased cellularity, and has a reduced viscosity so that it is often watery in consistency. Clot may form due to its increased content of fibrogen. The total cell count is usually raised above 3000 leucocytes $/ \mathrm{mm}^{3}$, and may exceed 50000 leucocytes $/ \mathrm{mm}^{3}$. Neutrophil polymorphs comprise over $50 \%$ of the cells present in fluids having high cell counts associated with active arthritis, but may comprise less than $50 \%$ of the cells in fluids having low cell counts during inactive phases of the disease. The content of albumin, globulin, IgA, IgG, IgM, and complement in the fluid is increased.

During acute episodes of crystal synovitis the fluid may be purulent in appearance, but the total and differential cell count is largely dependent upon the number of crystals present. Crystals of sodium urate (gout) and calcium pyrophosphate dihydrate (pseudogout) are identified by their specific optical properties.

In septic arthritis the total cell count may be quite low in the early stages and thus may easily be confused with an inflammatory arthropathy or crystal arthritis. In all doubtful cases, the presence of microorganisms is investigated using appropriate special stains and by culture.

In posttraumatic effusions red blood cells may only be numerous for a short time after the traumatic episode has occurred. Red blood cells may also be numerous due to the aspiration procedure, acute synovitis of any cause, haemophilia, or villonodular synovitis. Breaching of the articular surfaces following trauma or bone necrosis may be revealed by the presence of numergus intracellular fat droplets following ithe uptake of bone marrow fat by synopial fluid leucocytes.

\section{Platelet Aggregation with Ristocetin}

JANE HUGHES, ELAINE WILSON, T. $\mathcal{Q}_{\mathrm{v}}$. BARROWCLIFFE, AND PENELOPE STABLEFORTH (The Royal Free Hospital, Londen) Following the reports of Howard ford Firkin (1971, 1973) that Ristocepin induced platelet aggregation was valuable diagnostic test for $\Phi_{n}$ Willebrand's disease (vWd), 30 م viously diagnosed von Willebrand patients were reinvestigated. Standard in gations of blood coagulation and plitetet function were carried out, as well a as measurement of the factor VIII-relafed protein and Ristocetin-induced aggegation. With a final Ristocetin concentration of $1.0 \mathrm{mg} / \mathrm{ml}$, aggregation of von Willebrand platelet-rich plasma (PRP) reduced in all patients (less than $1 \bar{\Theta}^{\circ} \%$ aggregation compared with the control range of $30-70 \%$ ). In 29 out of 30 patients the findings supported the hypothesis that the von Willebrand factor is in the plasma, since Ristocetin-induced aggregation of von Willebrand platelets fesuspended in normal (N) platelet peor plasma (PPP) was normal, while aggregation of N-platelets in von Willebrand PPP was abnormal. The exception to this was patient 19, in whom mixilug experiments indicated a platelet defet (see table).

Twenty-eight patients had reduced or absent levels of factor VIII related protein, as reported by Zimmermath, Ratnoff, and Powell (1971).

Since the two patients with normal levels of VIII-related protein, cases 7 and 19 , had hereditary patterns c\&nsistent with an autosomal dominant inheritance, they were dissimilar from the cases of von Willebrand's disease whth 